\title{
Prognosis of Diabetics with Diabetes Onset before the Age of Thirtyone
}

\author{
II. Factors Influencing the Prognosis \\ T. Deckert, J. E. Poulsen, and M. Larsen \\ The Steno Memorial Hospital and the Copenhagen County Hospital, Data Processing Department, Gentofte, Denmark
}

Summary. In 307 patients with diabetes mellitus, developed prior to 1933 and before age 31 it was demonstrated that: (1) frequent contact with a specialized diabetes clinic from an early stage of the disease; (2) a good quality of "metabolic control"; (3) a low insulin dose; (4) a body weight of $10 \%$ less than ideal; and (5) a mean blood pressure below $100 \mathrm{~mm} \mathrm{Hg}$, all had significantly beneficial effects upon the survival. It was also found that patients domiciled in Copenhagen had a significantly better prognosis than patients domiciled outside Copenhagen. Frequent contact with a diabetes centre was accompanied by an appreciable decrease in disabling late diabetic complications.

Key words: Prognosis, juvenile diabetes, insulin, supervision, quality of regulation, body weight, blood pressure, domicile.

Juvenile diabetes (defined here as insulin-dependent diabetes diagnosed before the age of 31 ) is a serious disease involving a considerable excess mortality despite insulin therapy, dietary restrictions, guidance, and supervision [1]. As shown in the previous paper [1] the excess mortality is due to the development of nephropathy and cardiovascular complications. In order to elucidate indirectly the claim of a causal relationship between metabolic control and prognosis [2], we have investigated the importance of contact with a specialized diabetes unit to survival in juvenile diabetes, assuming that frequent and longlasting contact between the diabetic patient and a diabetes centre might influence the quality of metabolic control and thus the prognosis. In addition, we tried to elucidate directly the prognostic impor- tance of the quality of metabolic control, the magnitude of the insulin dose (as an indirect mean of calculating the degree of B-cell insufficiency), per cent of ideal body weight, blood pressure, and early institution of insulin therapy.

\section{Material}

The material comprises 307 consecutive diabetics whose diabetes had been diagnosed prior to 1933 and before the patients were 31 years of age. The material was described in the previous paper [1].

\section{Method}

The patient's survival was assessed on the basis of two parameters: [1] Duration of diabetes and [2] relative survival, i.e. survival in relation to the general population of the same age and sex. The calculation was based upon the mortality rates per thousand for the comparable total population of Denmark given in the Danish Year Books of Statistics (Statistisk Aarbog).

The prognostic importance of contact between the patient and the specialist unit was studied [1] by comparing the survival of diabetics who had a varying frequency of contact with the Steno Memorial Hospital during the first twenty years of diabetes, [2] by comparing the influence of domicile upon the survival of diabetics referred to the Steno Memorial Hospital within their first fifteen years of disease and [3] by comparing the survival in diabetics who had been referred to the Steno Memorial Hospital after a diabetes duration of $0-15,16-25$, and more than 25 years.

[1] and [2]: All the patients had been admitted to the Steno Memorial Hospital within the first 15 years 
of their diabetes $(n=180)$. One group $(n=42)$ did not have further contact with the Hospital after the first admission. The second group $(n=83)$ had sporadic contact, and the third group $(\mathrm{n}=55)$ had close contact with the Hospital. Close contact was taken to mean that the patient had been admitted 5 or more times and/or seen more than twenty times in the Out-patient Clinic in the course of the first twenty diabetes years. Sporadic contact was taken to mean that the patient had been admitted 2-5 times and/or seen 1-20 times in the Out-patient Clinic within the first twenty diabetes years.

Domicile was divided into Greater Copenhagen, urban areas outside Copenhagen and rural districts.

We tried to elucidate the prognostic influence of the quality of control by comparing the survival in groups of patients in whom this quality differed. Two hundred and fourteen patients had been seen so many times that a reasonable assessment of the mean quality of control was possible. The quality of control was assessed blind from records of the out-patient post-prandial concentration of blood glucose in the forenoon, variations in the blood glucose concentration during admissions, 24-hour urinary glucose excretion at the out-patient visits and during admissions, the occurrence of acetone in the 24-hour urine, and the frequency of ketoacidosis.

The total estimate was quantitated into 3 degrees:

1. Always relatively well-controlled, i.e. never ketoacidosis, never acetonuria, rarely (less than 10\% of the measurements) glucosuria, post-prandial blood sugar fairly stable and rarely (less than $10 \%$ of the measurements) $>250 \mathrm{mg} / 100 \mathrm{ml}$ (Hagedorn, Norman-Jensen) $(\mathrm{n}=24)$.

2. Ketoacidosis occurred at times ( $\leq 3$ times), nearly constant glucosuria, rarely acetonuria, post prandial blood sugar fluctuating and not infrequently. $>250 \mathrm{mg} / 100 \mathrm{ml}(\mathrm{n}=174)$.

3. Ketoacidosis more than 3 times, acetonuria in more than $10 \%$ of measurements, nearly always marked glucosuria ( $>100 \mathrm{~g} /$ day), and blood sugar greatly fluctuating and often (more than $50 \%$ of the measurements) $>250 \mathrm{mg} / 100 \mathrm{ml}(\mathrm{n}=16)$.

Since we endeavoured to use the $24 \mathrm{~h}$ dose of insulin as an indirect and rough mean of calculating the degree of B-cell insufficiency other factors which might influence the daily insulin requirement were reduced as far as possible. Therefore, 24-hours insulin requirement $/ \mathrm{kg}$ bodyweight only was registered in patients who fulfilled the following conditions:

Age $>18$ years

non obese

Serum creatinine: (method of Jaffé) below $1.4 \mathrm{mg} / 100 \mathrm{ml}$ or blood urea (method of van Slyke) $<45 \mathrm{mg} / 100 \mathrm{ml}$
No liver disease

No diabetogenic drugs

Non-pregnant

No infection

Insulin treatment for more than one year

Hospitalization at Steno Memorial Hospital for diabetes regulation between 1950 and 1965 .

The period between 1950 and 1965 was chosen in order to reduce the influence of insulin antibodies and glucagon contamination of the insulin preparations as far as possible. Before 1950 impure amorphous protamine insulin preparations were used which contained small amounts of glucagon, and before 1950 insulin resistance (more than 100 I.U. daily) was seen at Steno Memorial Hospital occasionally. Since 1950 Danish NPH-insulin has been used in nearly all patients, which means rather highly purified, glucagon free, $85 \%$ porcine $-15 \%$ bovine insulin, and since 1949 no case of insulin resistance has been seen at the Hospital. After 1965 we gradually changed to the use of pure porcine $\mathrm{NPH}$-insulin, a change which influenced the formation of insulin antibodies and thereby the daily dose [3]. The insulin requirements of the last day of hospitalization was recorded, when the patient was on proper diet, without Somogyi-phenomenon and with physical activity imitating the usual habits.

Body weight was ascertained at all visits to the Steno Memorial Hospital. The mean of all values measured in the same patient while adult was expressed as the percentage of the ideal weight according to the tables of Natvig [4]. This calculation could be performed for two hundred and eighty-three patients. In the others it could not be carried out, either because they were children who had not been followed in the Steno Memorial Hospital after they grew up or because no measurement of height was available. Twentyfive patients were overweight (weight $\geqslant 115 \%$ of ideal weight), and thirtysix patients were underweight (weight $<85 \%$ of the ideal weight).

Body height was measured in more than $95 \%$ of the patients. Patients in whom the diabetes began before the age of fifteen and whose final height as adults was less than $93 \%$ of the mean height for adult men and women were designated as growthretarded. The mean final body height was calculated on the basis of repeated measurements over a number of years after the age of 20 .

Systolic and diastolic blood pressure was measured with a sphygmomanometer and expressed as the mean blood pressure (diastolic pressure + one-third of the difference between diastolic and systolic pressure). The blood pressure was determined repeatedly over a number of years in nearly all 

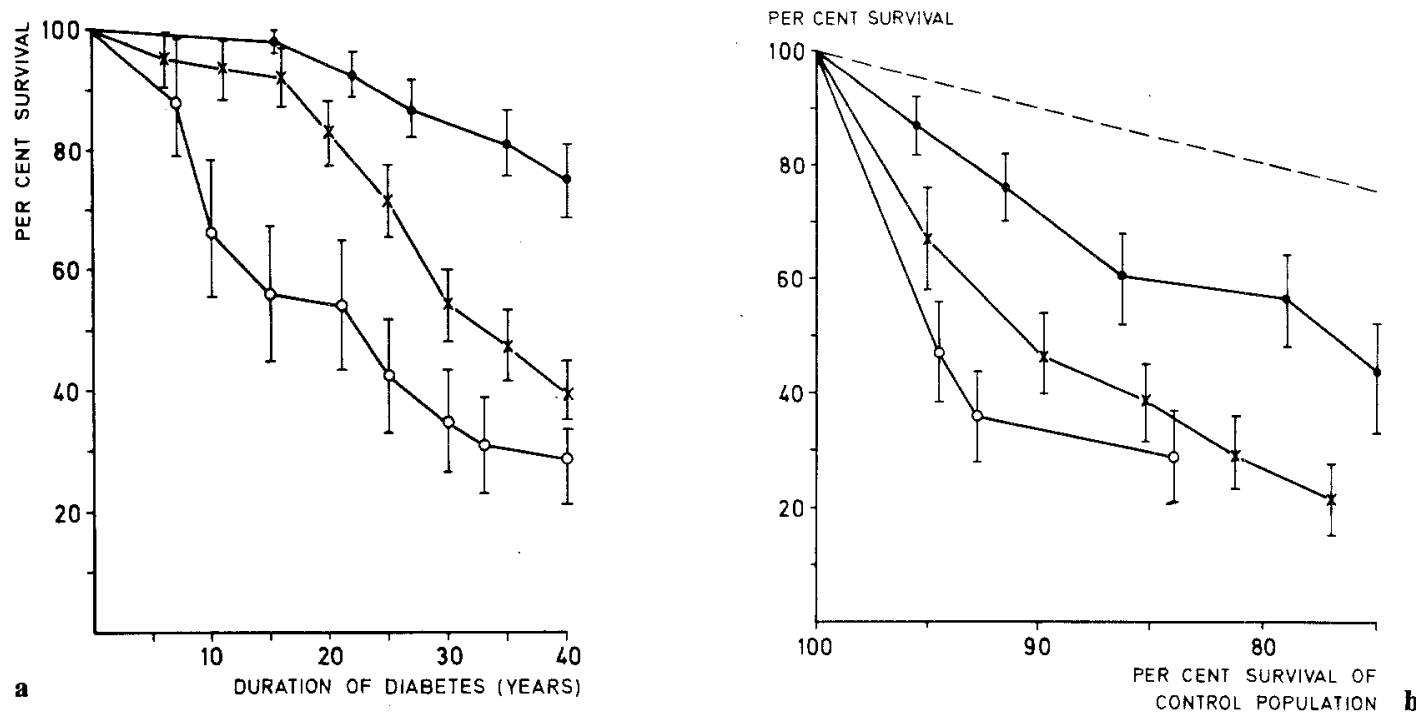

Fig. 1. Per cent survival (mean \pm SD) of 3 groups of juvenile diabetics until more than 40 years after onset of diabetes. The patients were admitted to the Steno Memorial Hospital within fifteen years from the onset of diabetes, and were in frequent $(-\ldots)(\mathrm{n}=55$ ), sporadic $(\times-\times)(n=83)$, or no contact $(\mathrm{O}-\mathrm{O})(\mathrm{n}=42)$ with the Hospital during the first twenty years of diabetes. a Duration of diabetes: Frequent contact/sporadic contact: $p=0.0001-$ Frequent contact/no contact: $p=0.0001-$ Sporadic contact/no contact: $p=n$. s. b Comparison with age- and sex-matched non-diabetics. Survival of the non-diabetics is shown by the dotted line: Frequent contact/ sporadic contact: $p<0.002-$ Sporadic contact/no contact: $p=n$. s. - Frequent contact/no contact: $p<0.0005$

adults. Blood pressure values were used only for patients who did not have proteinuria.

Only $55 \%$ of the patients had been started on insulin therapy at the time of diagnosis, but $95 \%$ were on insulin 5 years after diagnosis. In order to ascertain whether the treatment period without insulin at the beginning of the disease was of prognostic importance, a comparison was made of survival in patients whose insulin therapy had been started at diagnosis, in those in whom it was started within 2 years, and in those in whom it was started 3 or more years after diagnosis.

The definition of complications as blindness, proteinuria, uraemia, amputation, cerebral vascular insufficiency, myocardial infarction has been given previously [1].

In describing survival we have used decrement analyses [5] and for comparing the two decrement curves Gehan's Wilcoxon test $[6,7]$. These methods were used in a modification which pays regard to the full survival being unknown for part of the population and for entrance to the study being later than at the beginning of the studied lifetime [7].

Co-variation analyses were by the Spearman rank correlation, using the t-test, chi-square test, and Kruskal-Wallis test.

\section{Results}

Figure $1 \mathrm{a}$ and $\mathrm{b}$ illustrates the importance of contact between juvenile diabetics and a diabetes centre.
Table 1. Prevalence of complications (\%) in 151 patients $^{\mathrm{a}}$ admitted to the Hospital within fifteen years of the onset of diabetes and having frequent, sporadic, or no contact with the Hospital during the first twenty years of diabetes (minimal - maximal figures)

\begin{tabular}{lccc}
\hline & $\begin{array}{l}\text { Frequent } \\
\text { contact } \\
\mathrm{n}=51\end{array}$ & $\begin{array}{l}\text { Sporadic } \\
\text { contact } \\
\mathrm{n}=67\end{array}$ & $\begin{array}{l}\text { No } \\
\text { contact } \\
\mathrm{n}=33\end{array}$ \\
\hline $\begin{array}{lccc}\text { Diabetes duration (years) } \\
\text { as of 1.1.1973 (mean } \pm \mathrm{SD})\end{array}$ & $40 \pm 9$ & $32 \pm 11$ & $30 \pm 13$ \\
Blindness & $10-14$ & $19-38$ & $6-21$ \\
Proteinuria & $27-29$ & $39-55$ & $24-44$ \\
Uraemia & $12-20$ & $27-4$ & $12-41$ \\
Amputation/Gangrene & 10 & $8-22$ & $9-18$ \\
Cerebral vascular insult & 6 & $9-16$ & $6-15$ \\
Myocardial infarction & $27-31$ & $17-19$ & $12-18$ \\
\hline
\end{tabular}

a For twentynine patients no information regarding complications was available and in a further $5-10 \%$ the information was incomplete, especially in the no contact group. Therefore, minimal and maximal figures are given

Thus patients having frequent contact with the Steno Memorial Hospital during their first 20 years of diabetes had a significantly better prognosis than patients who had only sporadic or no contact after their first admission. The patients who were admitted to the Hospital after the first fifteen years of diabetes had a significantly shorter survival than any of the other groups $(\mathrm{p}<0.0001)$. There was also earlier and more frequent occurrence of serious late diabetic manifestations in patients having only sporadic con- 
Table 2. Duration of diabetes (years) at varying death rate (from Fig. 1 a)

\begin{tabular}{llll}
\hline Death rate & $\begin{array}{l}\text { Frequent } \\
\text { contact }\end{array}$ & $\begin{array}{l}\text { Sporadic } \\
\text { contact }\end{array}$ & $\begin{array}{l}\text { No } \\
\text { contact }\end{array}$ \\
\cline { 2 - 4 } & Years & Years & Years \\
\hline $5 \%$ & 19 & 6 & 2.5 \\
$10 \%$ & 24 & 17 & 6 \\
$15 \%$ & 29 & 19 & 7.5 \\
$20 \%$ & 36 & 22 & 8 \\
$25 \%$ & 40 & 24 & 9 \\
\hline
\end{tabular}

Table 3.

Per cent survival (mean $\pm \mathrm{SD}$ ) of two groups of juvenile diabetics receiving a daily insulin dose of $0.31-0.50$ or $\geq 0.51$ units $/ \mathrm{kg}$ compared to the per cent survival of age- and sex-matched nondiabetics. The difference between the two diabetic groups is significant $(p<0.05)$

\begin{tabular}{|c|c|c|}
\hline \multirow{2}{*}{$\begin{array}{l}\text { Age- and sex- } \\
\text { matched non- } \\
\text { diabetics }\end{array}$} & \multicolumn{2}{|c|}{$\begin{array}{l}\text { Diabetics } \\
\text { Insulin requirement (units/kg) }\end{array}$} \\
\hline & $\begin{array}{l}0.31-0.50 \\
(\mathrm{n}=29)\end{array}$ & $\begin{array}{l}>0.51 \\
(\mathrm{n}=103)\end{array}$ \\
\hline 95 & - & $90 \pm 4$ \\
\hline 90 & $94 \pm 6$ & $67 \pm 6$ \\
\hline 85 & $88 \pm 8$ & $60 \pm 6$ \\
\hline 80 & $69 \pm 11$ & $50 \pm 6$ \\
\hline
\end{tabular}

tact with the Hospital (Table 1). Relative survival (excess mortality) was at least twice as high $(400-500 \%)$ in patients having sporadic contact with the Steno Memorial Hospital as in patients having good contact $(100-200 \%)$ (Fig. 1b). Table 2 presents the mean duration of diabetes at times when the death rate was $5-25 \%$ in patients who had had, within their first twenty years of diabetes, frequent, sporadic, or no contact with the Steno Memorial Hospital after their first admission. Thus $10 \%$ of the patients who had frequent contact with the Hospital had died after twentyfour years of diabetes, while $10 \%$ of the patients with sporadic or no contact with the Steno Memorial Hospital were dead after 17 and 6 diabetes years respectively.

Figure $2 \mathrm{a}$ and $\mathrm{b}$ show that patients who contacted the Steno Memorial Hospital within the first fifteen years of diabetes and who were domiciled near the Hospital (in Greater Copenhagen) had a significantly better survival than patients living far away.

The one hundred and eighty patients who attended the Steno Memorial Hospital within the first fifteen years of their diabetes had a significantly better survival than sixty-four and sixty-three patients who contacted this Hospital between sixteen to twenty-five years or more than twenty-five years of diabetes duration respectively, although it is assumed that the survival of the latter groups during the first thirty years of diabetes was identical with
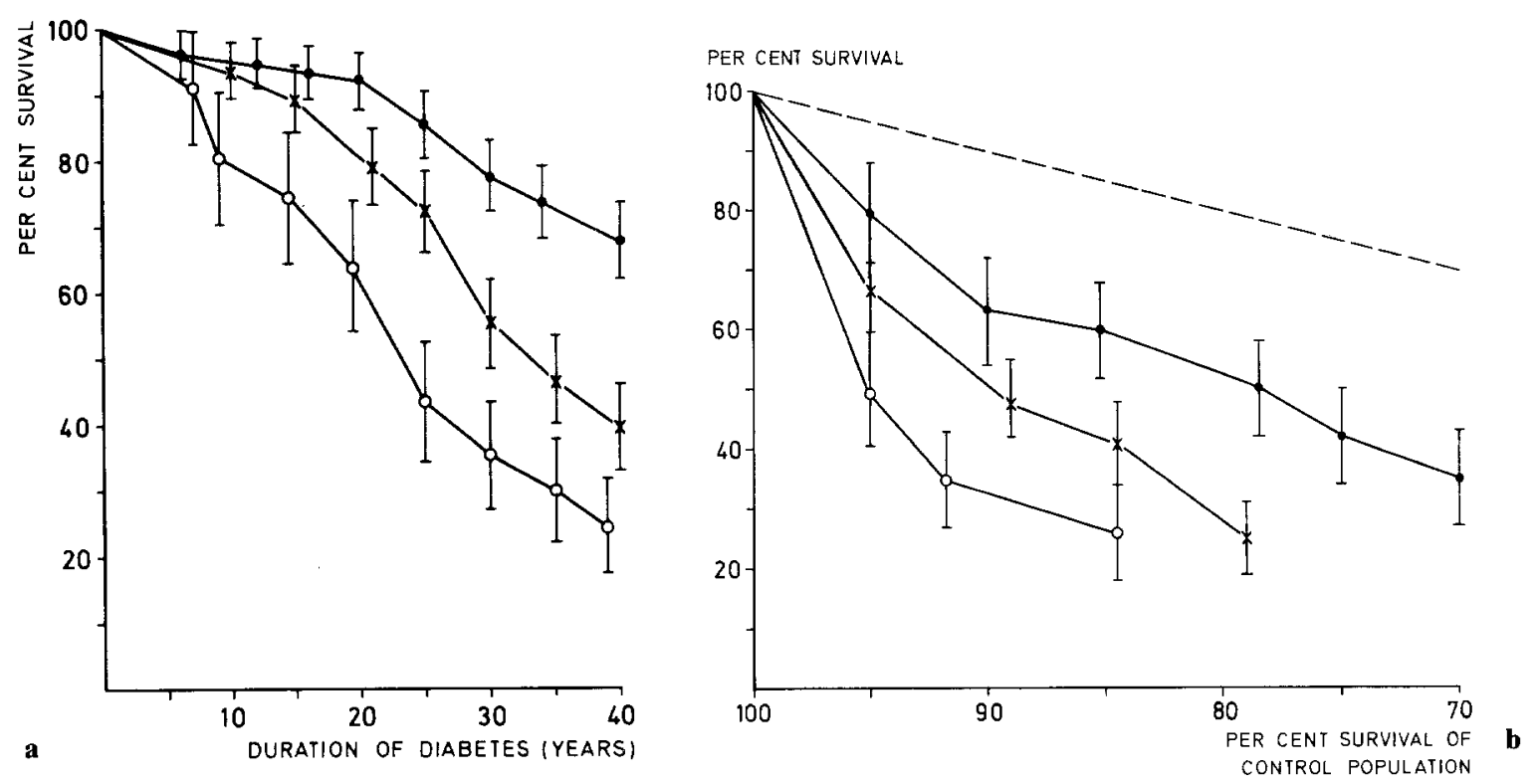

Fig. 2. Per cent survival (mean \pm SD) of 3 groups of juvenile diabetics living in Copenhagen $(\bullet-\bullet)(\mathbf{n}=80)$, provincial towns $(\times-\times)(n=61)$, or in rural districts $(\mathrm{O}-\mathrm{O})(\mathrm{n}=39)$. The patients were admitted to the Hospital during the first fifteen years of diabetes. a Duration of diabetes. Difference in survival time between the Copenhagen group and the other groups: Copenhagen/provincial towns: $\mathrm{p}<0.0005-$ Copenhagen/rural district: $\mathrm{p}<0.0001-$ Provincial town/rural district: $\mathrm{p}=\mathrm{n}$. s. b Comparison will age- and sexmatched non-diabetics. Survival of the non-diabetic population is shown by the dotted line: Copenhagen/provincial town: $p<0.001-$ Copenhagen/rural district: $\mathrm{p}<0.0001-$ Provincial town/rural district: $\mathrm{p}=\mathrm{n}$. s. 
that of patients who contacted the Hospital within the first fifteen years of diabetes $(p=0.024$ and 0.0034 , respectively).

If the patients are grouped by quality of metabolic control, relative survival was significantly better in the relatively well-controlled patients than in moderately or poorly controlled ones (Fig. 3) with the well-controlled group not differing significantly from the general population.

The same applied to patients treated with low doses of insulin, between 0.31 and 0.50 units $/ \mathrm{kg}$ body weight (Table 3 ). Only seven patients had a daily dose below 0.3 units $/ \mathrm{kg}$ body weight. Two of these patients could not be followed. Therefore, a statistical assessment of this group was omitted.

From Figure 4 it is apparent that patients with ideal body weight between 85 and $105 \%$ had a better relative survival than other patients. However, patients with markedly lowered ideal body weight had a much poorer survival.

Figure 5 indicates that patients whose mean blood pressure exceeded $100 \mathrm{~mm} \mathrm{Hg}$ fared worse than those with a lower mean blood pressure, even though the blood pressure measured in patients with diabetic nephropathy was not included in the calculation. Patients whose blood pressure is not listed (because of existing nephropathy or because it had not been measured) had a significantly poorer relative survival than the other 2 groups $(\mathrm{p}<0.0005)$.

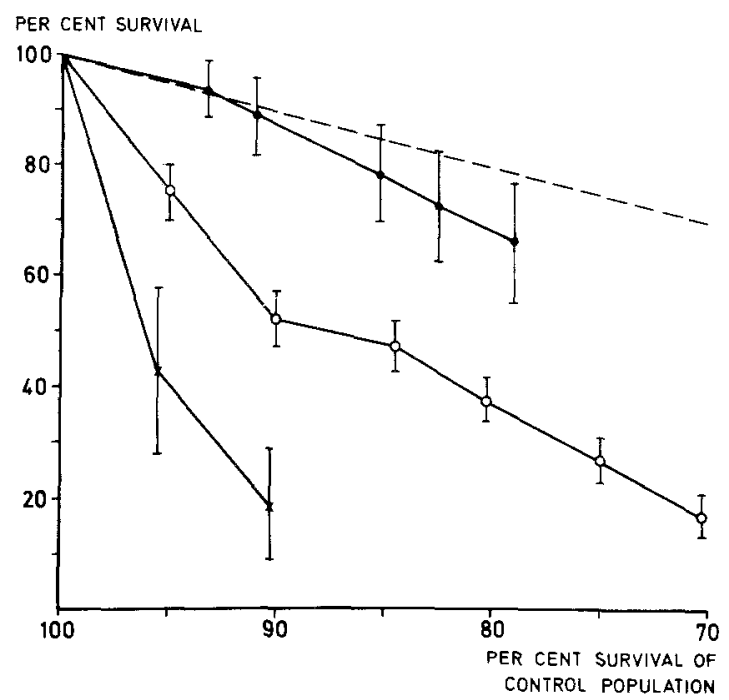

Fig. 3. Per cent survival (mean \pm SD) of three groups of juvenile diabetics who were relatively well controlled $(-0)(\mathrm{n}=24)$, moderately $(\mathrm{O}-\mathrm{O})(\mathrm{n}=174)$ or poorly controlled for many years $(\times-\times)(n=16)$, compared to the per cent survival of age- and sex-matched non-diabetics (dotted line). Difference in survival time between the groups: Well controlled/moderately controlled: $\mathrm{p}=0.01-$ Well controlled/poorly controlled: $\mathrm{p}=$ 0.001 - Moderately controlled/poorly controlled: $p=0.02$

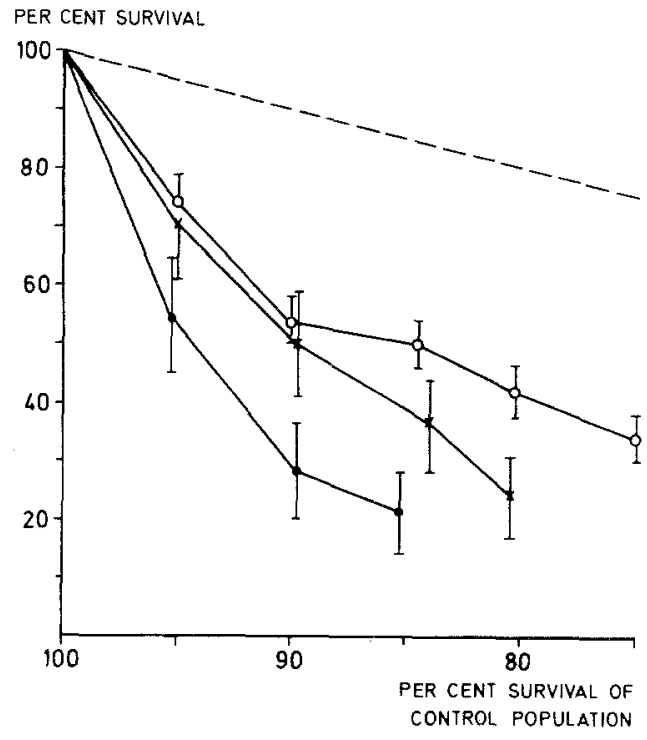

Fig. 4. Per cent survival (mean \pm SD) of three groups of juvenile diabetics of a body weight $\leqslant 84 \%(-3)(\mathrm{n}=38)$, $85-105 \%(\mathrm{O}-\mathrm{O})(\mathrm{n}=192)$, and $>105 \%(\times-\times)(\mathrm{n}=$ 53) of the ideal body weight compared to the per cent survival of age- and sex-matched non-diabetics (dotted line): $\leqslant$ 84/ $85-104 \%: \mathrm{p}<0.0005-\leqslant 84 / \geq 105 \%: \mathrm{p}=0.05-85-104 \% /$ $\geqslant 105: \mathrm{p}=0.085$

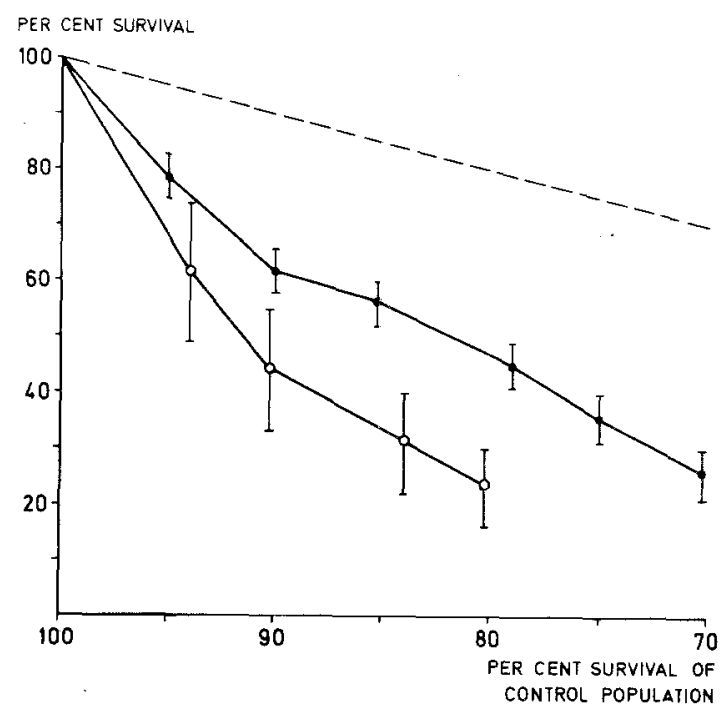

Fig. 5. Per cent survival (mean \pm SD) of 2 groups of juvenile diabetics with a mean blood pressure of $\leqslant 100 \mathrm{~mm} \mathrm{Hg}$ (_$(\mathrm{n}=184)$, and $>100 \mathrm{~mm} \mathrm{Hg}(\mathrm{O}-\mathrm{O})(\mathrm{n}=43)$ compared to the per cent survival of age- and sex-matched non-diabetics (dotted line). Difference between the two blood pressure groups ( $\mathrm{p}=$ 0.058 ) 
Of the one hundred and nineteen children who developed diabetes before the age of fifteen years, $16 \%$ were growth-retarded. However, there was no difference in survival between growth-retarded and non-growth-retarded diabetics $(\mathrm{p}=0.53)$. Statistical analysis of the total material showed a relationship between height and age at onset of diabetes, patients developing diabetes prior to the age of fifteen attaining a significantly lower final height than patients whose disease developed later.

The duration of the insulin-free period (number of years between diagnosis and institution of insulin therapy) was of no importance to survival in the present study $(p=0.3)$.

\section{Discussion}

The study revealed that patients having frequent contact with the Steno Memorial Hospital fared better than those having only sporadic or no contact with the Hospital after their first admission.

The explanation for this difference lies probably not in a selection of patients because of early death, since there was still a significantly better survival among patients in frequent contact with the Steno Memorial Hospital, even when patients who died within the first fifteen years of diabetes were excluded from the groups (the first contact with the Steno Memorial Hospital was an average of 7.5 years after the onset of diabetes).

Analysis of the 3 groups of patients (frequent contact, sporadic contact, and no contact during the first twenty years of diabetes) showed that the groups were comparable as regards age, sex, onset of diabetes, retardation of growth, insulin-free years, blood pressure, dose of insulin per $\mathrm{kg}$ body weight, and quality of control. On the other hand, there were significantly fewer deviations from the optimal body weight $(85-105 \%$ of the ideal weight $)(p<0.05)$, a significantly larger number of Copenhagen residents $(\mathrm{p}<0.0001)$, and a significantly shorter duration between diagnosis and first admission to the Steno Memorial Hospital (viz. 6.4, 8.0, and 9.1 years) $(p=$ 0.0025 ) in the group of patients who had kept up a frequent contact with the Steno Memorial Hospital. This is hardly surprising, as the Steno Memorial Hospital is situated in Copenhagen and has always tried to keep body weight of diabetic patients $10 \%$ below the ideal weight.

Patients domiciled in Copenhagen proved to have a better prognosis than patients domiciled in other parts of Denmark. Since the Copenhagen non-diabetic population has a poorer survival than the rural Danish population [8], the explanation of the sig- nificantly better relative survival of juvenile diabetics domiciled in Copenhagen must be presumed to be that diabetics in Copenhagen have better access to diabetes therapy and supervision than patients domiciled in other parts of the country. Dietary supervision may also have been better.

The explanation of the excess mortality among patients having only sporadic contact with the Steno Memorial Hospital is primarily an earlier and more common occurrence of uraemia (Table 1). Blindness and stroke are also more common in the sporadically supervised group of diabetics. This difference is increased when considering that patients seen frequently showed a considerably longer duration of diabetes than did the other groups. The values found for myocardial infarction carry a marked uncertainty, as it was not possible to analyse accurately in retrospect silent infarcts or infarcts which were not followed by angina pectoris or cardiac failure. The values in the group of patients who did not attend again during the first twenty years of their diabetes (no supervision) are even more uncertain, as the group is small and information concerning complications deficient in $20 \%$.

It is not possible to prove that patients having frequent contact with the Steno Memorial Hospital during their first twenty years of diabetes had a better quality of metabolic control than patients seen rarely. However, it must be assumed that patients who frequently attend the Out-patients' Clinic acquire a more intimate knowledge of their disease than do others and that in such cases it is possible to avoid lengthy periods of poor regulation giving rise to few symptoms. In the present study a rough estimate of the quality of control was made without knowing the patients' prognosis. Although this method of assessing control is questionable twenty-four out of two hundred and twelve patients appeared to be relatively well-controlled. However, it is uncertain whether the relatively good control in these few cases was a result of the efforts made by the patients and doctors, or due to another type of diabetes, e.g. a type with a preserved, though slight endogenous insulin production. At least, there was a significant correlation between the insulin dose $/ \mathrm{kg}$ body weight and the quality of control $(p<0.01)$. As a low dose of insulin may reflect preserved endogenous insulin production [9], and as preserved endogenous insulin production in most cases greatly facilitates the control [10], the correlation may be due to this cause. The correlation between insulin dose $/ \mathrm{kg}$ body weight and control quality is presumably also the explanation of the better prognosis in patients receiving a low dose of insulin (Table 3). Goodkin [11] also found patients treated with low doses of insulin to have a 
better prognosis than patients treated with high doses. However, Goodkin's material was not representative of juvenile diabetes. The explanation of a better relative survival among patients having a body weight $85-105 \%$ of the ideal, compared with patients of lower or higher body weight, is not clear. However, it cannot be ruled out that patients who kept their weight at the lower end of normal obtained this by a more ascetic way of living which has benefited their diabetes control. The group of patients whose body weight was more than $105 \%$ of the ideal weight is, of course, loaded by the twentyfive patients whose body weight exceeded $115 \%$ of the ideal weight.

Hypertension is well-known as a risk factor. Goodkin [11] also demonstrated that hypertension aggravates the prognosis in diabetics, but in his study patients with nephropathy had not been excluded. Essential hypertension without proteinuria was rare in the juvenile diabetics. Only seven out of two hundred and twenty-seven (3\%) had a mean blood pressure of $\geq 115 \mathrm{~mm} \mathrm{Hg}$. This is in contrast to the common occurrence of hypertension in patients with maturity-onset diabetes.

That patients developing diabetes before the age of fifteen grow less than non-diabetics or diabetics who develop their disease after the age of fifteen is well-known [12], and also confirmed in the present study.

In conclusion it seems advisable to motivate juvenile diabetics for regular visits at a specialized diabetes clinic from the onset of the disease, to keep body weight and blood pressure within normal limits and to achieve metabolic regulation as good as possible.

\section{References}

1. Deckert, T., Poulsen, J.E., Larsen, M.: Prognosis of diabetics with diabetes onset before the age of thirtyone. I: Survival, cause of death, and complications. Diabetologia 14, 359-362 (1978)

2. Pirart, J.: Diabète et complications degénératives. Diabete Metab. 3, 97-107, 173-182, 245-256 (1977)

3. Deckert, T., Andersen, O. O., Poulsen, J.E.: The clinical significance of highly purified pig-insulin preparations. Diabetologia 10, 703-708 (1974)

4. Natvig, H.: Nye høyde og vekttabeller for norske kvinner og menn. Oslo: Landsforeningen for Kosthold og Helse 1956

5. Kaplan, E. L., Meier, P.: Nonparametric estimation from incomplete observations. T. Am. Statist. Assoc. 53, 475-481 (1958)

6. Gehan, E.: A generalized Wilcoxon test for comparing arbitrary single censored samples. Biometrica 52, 203-223 (1965)

7. Aalen, O.: Nonparametric inference for a family of counting processes. Ann. Statist. (In Press) (1978)

8. Horwitz, O., Weber, J.: Ægteskabelig stilling og dødelighed. Ugeskr. Læger. 22, 1089-1104 (1973)

9. Eff, Chr., Faber, O., Deckert, T.: Insulin secretion in long term juvenile diabetics with low insulin requirement measured by C-peptide immunoreactivity. Diabetologia (In press)

10. Grajwer, L. A., Pildes, R. S., Horwitz, D. L., Rubenstein, A. H.: Control of juvenile diabetes mellitus and its relationship to endogenous insulin secretion as measured by C-peptide immunoreactivity. J. Pediatr. 90, 42-48 (1977)

11. Goodkin, G.: Mortality factors in diabetes. J. Occup. Med. 17, $716-721$ (1975)

12. Petersen, H., Draminsky, B.K., Deckert, T., Nielsen, E.: Growth, body weight and insulin requirement in diabetic children. Acta Paediatr. Scand. (In Press) (1978)

Received: July 22, 1977,

and in revised form: January 18,1978

Torsten Deckert

Steno Memorial Hospital

Niels Steensens Vej 2

DK-2820 Gentofte

Denmark 